\title{
Contenido de Ácido Fítico y Fósforo Inorgánico en Vigna unguiculata y Phaseolus vulgaris Germinadas a Diferentes Temperaturas
}

\author{
Lilí Rodríguez-Blanco ${ }^{(1)^{*}}$, Bernardo Lucas-Florentino ${ }^{(2)}$, Edith Miranda-Cruz ${ }^{(1)}$ y Martha Guerrero- \\ Olazarán ${ }^{(3)}$ \\ (1) Universidad Juárez Autónoma de Tabasco, División Académica de Ciencias Agropecuarias, Cuerpo \\ Académico Biotecnología y Calidad Alimentaria. Centro, 86298, Tabasco, México. \\ (e-mail: Iyrodblanc@gmail.com; lili.rodriguez@ujat.mx). \\ (2) Universidad Nacional Autónoma de México, Facultad de Química. Circuito exterior S/N, Coyoacán, 04510, \\ Ciudad de México, CDMX. \\ (3) Universidad Autónoma de Nuevo León, Facultad de Ciencias Biológicas, Instituto de Biotecnología. \\ Ciudad Universitaria, 66450, San Nicolás de los Garza, N.L., México
}

*Autor a quien debe ser dirigida la correspondencia

Recibido Ago. 23, 2017; Aceptado Oct. 23, 2017; Versión final Dic. 24, 2017, Publicado Abr. 2018

\begin{abstract}
Resumen
El objetivo de este estudio fue evaluar las condiciones de germinación en tiempo y temperatura, para la disminución del ácido fítico en Vigna unguiculata y Phaseolus vulgaris. Se analizó el contenido de ácido fítico, fósforo inorgánico y fósforo total, bajo distintas condiciones de temperatura y tiempo de germinación. Las condiciones óptimas de germinación se establecieron a $21^{\circ} \mathrm{C}$ entre dos a cuatro días. La variedad corazón negro de Vigna unguiculata, presentó el mayor porcentaje de disminución del ácido fitico a los dos días de germinación a $21^{\circ} \mathrm{C}$, con 93,75 a 94,85\%, y el mayor contenido de fósforo inorgánico a los seis días de germinación a $29^{\circ} \mathrm{C}$, con 23 veces de aumento. Se obtuvo harinas mejoradas nutricionalmente, con una mayor biodisponibilidad de fósforo inorgánico y de los nutrientes ligados a él, factibles de ser utilizadas en la producción de alimentos para diferentes grupos de la población.
\end{abstract} germinación.

\section{Content of Phytic Acid and Inorganic Phosphorus in Vigna unguiculata and Phaseolus vulgaris Germinated at Different Temperatures}

\begin{abstract}
The evolution of the concentration of phytic acid, inorganic phosphorus and total phosphorus in Vigna unguiculata and Phaseolus vulgaris according to different conditions of temperature and time of germination was studied. The optimum conditions of germination were established at $21^{\circ} \mathrm{C}$ between 2 to 4 days. The variety Vigna unguiculata black heart presented the higher reduction of phytic acid at $21^{\circ} \mathrm{C}$, with 93,75 to $94,85 \%$ decrease in two days, and in the content of inorganic phosphorus at $29^{\circ} \mathrm{C}$, with 23 times of increase in six days of germination. The results suggest a higher bioavailability of inorganic phosphorus and nutrients linked to it, generating legumes with higher nutritional value, which could be used in the production of improved foods for different population groups.
\end{abstract}

Keywords: phytic acid; inorganic phosphorus; Vigna unguiculata; Phaseolus vulgaris; germination temperature. 


\section{INTRODUCCIÓN}

Las legumbres juegan un papel importante en la dieta del ser humano, constituyen la fuente principal de proteína vegetal y minerales en la población vulnerable del mundo (Marrugo-Ligardo et al., 2016). No obstante, pese a su gran valor nutricional, las legumbres contienen también algunos tóxicos naturales, que por sus propiedades actúan como compuestos antinutritivos (Gonçalves et al., 2016); uno de los más importantes relacionado con la salud y nutrición humana, es el ácido fítico (ácido mioinositol hexafosfórico o Ins $\mathrm{P}_{6}$ ) (Kumar et al., 2010;). El ácido fítico (AF) es un metabolito termoestable, que constituye la forma de almacenamiento primario de fosforo en vegetales, en legumbres su contenido varía de 0.14 a $2.05 \%$, representando del 18 al $88 \%$ del fósforo total en granos y semillas, distribuido uniformemente en los cotiledones y eje embrionario de las legumbres (Reddy et al., 1982). El contenido de AF en legumbres es muy variable, incluso dentro de la misma especie; esta variación puede deberse a la especie, variedades, localización, condiciones de irrigación, tipo de suelo, clima, periodo del año de la cosecha, entre otros factores (Gonçalves et al., 2016; Rijal y Adhikari, 2012).

La estructura única del AF le proporciona la capacidad de quelar fuertemente varios cationes, principalmente $\mathrm{Ca}, \mathrm{Mg}$, Fe y $\mathrm{Zn}$, formando sales insolubles llamadas fitatos; que generan un efecto adverso en la absorción intestinal de estos minerales en el ser humano ocasionando su deficiencia; impactan también negativamente, en la utilización de proteínas y lípidos; aspectos de gran importancia, sobre todo en los individuos que dependen nutricionalmente de alimentos vegetales (Kumar et al., 2010).

El fósforo, juega un papel relevante en diversos procesos metabólicos vitales para todos los organismos vivos, como formar parte de estructuras macromoleculares, en la generación de energía y en la regulación metabólica (Hegeman y Grabau, 2001), de ahí la importancia de su disponibilidad en forma libre como fósforo inorgánico (Pi), al cual puedan acceder las células de los organismos, así como a los otros nutrientes que se encuentran quelados. En el ser humano, el fósforo es ingerido como componente natural en alimentos de origen animal (como un constituyente de fosfoproteínas, fosfolípidos de membrana, ATP, ADP, ADN, ARN), y vegetal como ácido fítico o fitatos, así como aditivo en alimentos procesados industrialmente. El fósforo de origen animal es absorbido en el intestino como fósforo inorgánico, por arriba del $80 \%$, la biodisponibilidad del fósforo de origen vegetal (fitatos) es muy baja (menor al $40 \%$ ); mientras que, la absorción intestinal del fósforo inorgánico procedente de conservadores en los alimentos procesados industrialmente es del $100 \%$ (Karp et al., 2012).

Los fitatos se acumulan en las semillas durante el periodo de maduración, y es la principal forma de almacenamiento de fosfato, mioinositol y minerales durante la germinación y crecimiento de la nueva planta. El fósforo en esta forma no es utilizado por ningún organismo monogástrico, debido a que carecen de la enzima degradadora de fitato o fitasa (Olukosi, 2012), encargada de llevar a cabo la hidrólisis del AF o del fitato, y liberar fósforo inorgánico, derivados de mioinositol, y los nutrientes quelados (minerales, proteínas, carbohidratos o lípidos), para su mejor aprovechamiento biológico. En las legumbres, las fitasas se localizan en los cotiledones (Hegeman y Grabau, 2001). Estudios en polen y semillas de plantas indican que hay dos tipos de fitasas: una constitutiva y otra inducible por la germinación, ambas tienen que ver con la hidrólisis del AF durante la germinación. La fitasa constitutiva inicia la hidrólisis durante los estadios primarios de la germinación, como el remojo previo (Greiner et al., 2007), mientras que la fitasa inducible es sintetizada de novo durante la germinación a través de un ARNm preexistente (Jackson y Linskens, 1982). La fitasa inducible actúa sobre los fitatos permitiendo que el fósforo inorgánico y minerales, resulten accesibles para el desarrollo y crecimiento de la planta (Greiner et al., 2007).

Los estudios desarrollados sobre la disminución del AF en semillas, en beneficio de la nutrición de organismos monogástricos y del medio ambiente, han seguido dos caminos para lograr el mismo objetivo: el enfoque transgénico y el enfoque no transgénico (Rostami y Giri, 2013). En caso del presente trabajo, aporta conocimiento al enfoque no transgénico. El objetivo de este estudio fue evaluar las condiciones de germinación en tiempo y temperatura, para la disminución del ácido fítico en Vigna unguiculata (frijol pelón) y Phaseolus vulgaris (frijol negro), dos legumbres de gran consumo en México (Lagunes-Espinoza et al., 2008).

\section{MATERIAL Y MÉTODOS}

Considerando que los organismos monogástricos no son capaces de aprovechar el fósforo del AF, por carecer de fitasas, que el AF es soluble en agua, y la presencia de fitasas endógenas (constitutivas e inducibles) en las legumbres; se definieron condiciones experimentales para la activación de las fitasas endógenas, mediante el remojo ácido y la germinación a diferentes temperaturas durante seis días; tiempo suficiente para la síntesis de las fitasas vegetales (Hegeman y Grabau, 2001).

La actividad de fitasa en las legumbres es menor que en cereales, excepto en soya. La temperatura afecta la actividad de fitasa en diferentes semillas de legumbres como haba, lentejas y frijol negro, la cual disminuye 
gradualmente a partir de los $50{ }^{\circ} \mathrm{C}$, e incluso a partir de los $45^{\circ} \mathrm{C}$ en chícharo (Abdel-Gawad et al., 2013). Para la obtención de las muestras de harinas a evaluar, las semillas se someterán a un proceso de secado a $55 \pm 2{ }^{\circ} \mathrm{C}$ durante $48 \mathrm{~h}$, lo cual puede afectar la estabilidad de la actividad de fitasa. Por lo anterior, se planteó evaluar los productos de la actividad de fitasa durante el proceso de germinación: los contenidos de Pi y ácido fítico, principales indicadores de la acción de las fitasas endógenas, con la finalidad de elegir las mejores condiciones de tiempo y temperatura en la disminución de AF en frijol negro y dos variedades locales de frijol pelón, legumbres de gran consumo en México (Lagunes-Espinoza et al., 2008).

Las legumbres Phaseolus vulgaris y dos variedades locales de Vigna unguiculata, ombligo café y corazón negro, se adquirieron en el Municipio de Comalcalco, Tabasco, México. Se trasladaron al Laboratorio de Biotecnología de Alimentos, de la División Académica de Ciencias Agropecuarias, de la Universidad Juárez Autónoma de Tabasco. Las semillas se seleccionaron, y se almacenaron utilizando técnicas tradicionales (Lagunes-Espinoza et al., 2008), para su posterior utilización. Todos los materiales utilizados en la determinación de fósforo inorgánico y fósforo total se trataron para asegurar que estuvieran libres de fósforo. Los reactivos utilizados, fueron grado reactivo.

\section{Diseño experimental}

Se utilizó un diseño de bloques completos al azar con un arreglo factorial $3 \times 4$, con doce tratamientos. El diseño de tratamientos consistió en un arreglo factorial de los factores: Tiempo de germinación con cuatro niveles $\left(0,2,4\right.$ y 6 días), y la temperatura de germinación con tres niveles $\left(21,25\right.$ y $\left.29^{\circ} \mathrm{C}\right)$; considerándose el bloqueo del factor especie de las legumbres que se emplearon en este estudio: Phaseolus vulgaris y las dos variedades de Vigna unguiculata. El nivel de significancia se fijó en $p<0,01$. Los datos obtenidos se analizaron mediante un ANOVA utilizando el software SAS, V6.0 para Windows, se realizaron comparaciones múltiples de medias de acuerdo con el procedimiento de Tukey. Los resultados se presentan como la media \pm la desviación estándar de tres determinaciones.

\section{Germinación}

Las condiciones de remojo y tiempo de germinación se establecieron en base a los hallazgos de Hegeman y Grabau (2001) para la fitasa expresada en soya. Previo a los ensayos experimentales, las semillas limpias se desinfectaron con una solución de hipoclorito de sodio al $1 \%$ durante media hora. Posteriormente se hicieron lavados consecutivos con agua destilada, hasta alcanzar pH neutro (Díaz et al., 2007). Las semillas limpias se sometieron a un proceso de remojo ácido (ácido cítrico $1 \%$ ) durante 18 a $20 \mathrm{~h}$ a temperatura ambiente (TA), utilizando una relación semilla: agua de $1: 3(\mathrm{p} / \mathrm{v})$, posteriormente se drenaron, y se pasaron a cajas Petri con papel absorbente comercial, para iniciar el proceso de germinación a tres temperaturas diferentes $(21,25$ y $29^{\circ} \mathrm{C}$ ) en oscuridad, con 70 a $80 \%$ de humedad, durante 0, 2, 4 y 6 días. Durante el proceso de germinación las semillas fueron hidratadas por aspersión, con una solución de ácido cítrico al $1 \%$ cada $12 \mathrm{~h}$. Terminados los tiempos de germinación de cada leguminosa, las semillas se secaron a $55 \pm 2{ }^{\circ} \mathrm{C}$ durante $48 \mathrm{~h}$, se dejaron enfriar a TA y se molieron. Los germinados molidos se pasaron a través de un tamiz de malla 100 para obtener la harina; la cual se guardó en frascos de vidrio a temperatura ambiente, hasta su uso.

\section{Determinación de ácido fítico}

La determinación de AF se realizó según el método de Frühbeck, validado por Sotelo et al. (2002) en legumbres, que determina el valor real del ácido fítico ( $\operatorname{lns}_{6}$ e InsP $\mathrm{P}_{5}$ ), ya que estos derivados poseen la capacidad de formar complejos principalmente con minerales, no así los otros derivados del inositol fosfato, con cuatro o menos grupos fosfato (Abdel-Gawad, 2016). La harina de legumbres desgrasada (menos del 5 $\%$ de grasa), se extrajo con HCL 0,65 N. El pH de la mezcla se ajustó entre 0 y 1,0. La mezcla se sometió a una agitación vigorosa durante dos $\mathrm{h}$ a TA. El extracto obtenido se centrifugo a $17000 \mathrm{~g}$ durante $30 \mathrm{~min}$. Se colectó el sobrenadante, y se tomó una alícuota que se diluyó con agua desionizada. Se recomienda la dilución 1:25 para alimentos que contengan $1 \%$ o más de AF, y la dilución 5:25 para contenidos menores. El pH se ajustó a un valor de 6,0 con solución $\mathrm{NaOH} 1 \mathrm{~N}$ y luego se tomaron $10 \mathrm{~mL}$ de la alícuota diluida, que se transfirieron a una columna de resina de $8 \mathrm{~mm} \times 65 \mathrm{~mm}$ (AGI-X8, 200-400 mesh, 0,5 g, Bio Rad No. 140$1451 \mathrm{La}$ columna se lavó con $15 \mathrm{~mL}$ de $\mathrm{NaCl} 0,1 \mathrm{~N}$. El ácido fítico se eluyó con $15 \mathrm{~mL}$ de $\mathrm{NaCl} 0,7 \mathrm{~N}$ y se recolectó el extracto purificado. Se tomaron $3 \mathrm{~mL}$ de agua desionizada (blanco), o bien $3 \mathrm{~mL}$ de los estándares de soluciones de fitato de sodio (Sigma Co.), cuyo contenido fue de 5 a $50 \mu \mathrm{g} / \mathrm{mL}$ en agua desionizada), o bien los extractos purificados a través de la columna a los que previamente se les ajustó el pH a 3 y se les adicionó $1 \mathrm{~mL}$ del reactivo de Wade: $0,03 \%$ de $\mathrm{FeCl}_{3} 6 \mathrm{H}_{2} \mathrm{O}$ (Sigma Co.), más $0,3 \%$ de ácido sulfosalicílico disueltos en agua desionizada. Después de la agitación durante $5 \mathrm{~s}$ se leyó la absorbancia a $500 \mathrm{~nm}$. Los resultados se expresaron en $\mathrm{g}$ de ácido fítico/100 $\mathrm{g}$ de semilla germinada. 


\section{Determinación de fósforo inorgánico}

Previo al análisis de fósforo, se llevaron a cabo cinéticas de extracción para determinar el tiempo de extracción más adecuado para cada temperatura evaluada, se utilizaron las muestras del tiempo cero y seis días de germinación en las tres legumbres, a las tres temperaturas de germinación evaluadas. Se consideraron los tiempos de extracción en las diferentes temperaturas de germinación, que fueron de $24 \mathrm{~h}$ para las muestras germinadas a $21^{\circ} \mathrm{C}, 4 \mathrm{~h}$ para las de $25{ }^{\circ} \mathrm{C}$ y $8 \mathrm{~h}$ para las de $29 \stackrel{\circ}{\circ} \mathrm{C}$. El fósforo inorgánico fue determinado por el método descrito por Wilcox et al. (2000). Cada vez que se realizaron los análisis se construyó una curva estándar para fósforo, mediante concentraciones conocidas de fosfato mono-potásico $\left(\mathrm{KH}_{2} \mathrm{PO}_{4}\right)$, y el reactivo colorimétrico ( $1 \mathrm{~g}$ de ácido ascórbico, ácido sulfúrico $6 \mathrm{~N}$, y molibdato de amonio 2,5\%). Se pesaron $50 \mathrm{mg}$ de la harina de cada legumbre, se transfirieron a un tubo para su análisis (triplicado). A cada muestra se le agregó $1,0 \mathrm{~mL}$ de agua desionizada, se agitó de 5 a 7 min hasta su disolución. Se incubó a $25^{\circ} \mathrm{C}$, a 14000 rpm. Pasado el tiempo de extracción para cada legumbre y temperatura de germinación, se le agregó 1,0 mL de ácido tricloroacético al $15 \%$ y se agitó por 15 min en forma intermitente. Posteriormente se centrifugó a 8 $000 \mathrm{~g}$ por $30 \mathrm{~min}$ a temperatura ambiente. El sobrenadante obtenido se pasó a un tubo limpio y se centrifugó de nuevo a $14000 \mathrm{~g}$ por $10 \mathrm{~min}$. Del sobrenadante final se tomaron $200 \mu \mathrm{L}$, se colocó en un tubo de $2 \mathrm{~mL}$, se le agregó $800 \mu \mathrm{L}$ del reactivo colorimétrico y se incubó por 40 minutos a $37 \stackrel{\circ}{\mathrm{C}}$, posteriormente la solución incubada se transfirió a celdas de poliestireno, para leer su absorbancia a $820 \mathrm{~nm}$. Los resultados se expresaron como $\mathrm{mg}$ de $\mathrm{Pi} / \mathrm{g}$ de semilla germinada.

\section{Determinación de fósforo total}

El análisis de fósforo total (Pt) se realizó según las metodologías de Shelton y Harper (1941). Cada vez que se realizaron determinaciones de Pt se preparó una curva de calibración de fósforo. Para ello, se preparó un primer estándar de fósforo de 200 ppm, a partir de éste se preparó un segundo estándar de 50 ppm de fósforo, de este último estándar se preparan las concentraciones de la curva de calibración, que fueron de 0, 2, 4, 8, 12, y 16 ppm de fósforo. Los resultados se expresaron como $\mathrm{mg}$ de Pt/100 g de semilla germinada.

\section{RESULTADOS Y DISCUSIÓN}

Los resultados se presentan en dos subsecciones: una en la que se muestra y analiza el contenido de ácido fítico y una segunda en que se muestra y discute el comportamiento del contenido de fósforo (Pi y Pt) en las legumbres evaluadas.

\section{Ácido fítico}

En la Tabla 1, se observa como el contenido de ácido fítico en las legumbres estudiadas, disminuye de forma importante conforme avanza el proceso de germinación, y presenta diferencias en las temperaturas analizadas $(p<0,05)$. La mejor temperatura de germinación para las tres legumbres fue a $21^{\circ} \mathrm{C}$, donde las dos variedades de Vigna unguiculata presentaron el contenido de AF más bajo a los dos días de germinación, la variedad corazón negro presentó un 94,85 \% de reducción de AF, le siguió la variedad ombligo café con un $93,75 \%$, quien a su vez a los cuatro y seis días de germinación, ya no presentó valores de AF detectables $\left(^{*}\right.$ ) en ninguna de las tres temperaturas analizadas. Mientras que, Phaseolus vulgaris presento sus valores más bajos a los cuatro días de germinación, correspondientes a un $81,18 \%$ de reducción de AF.

La germinación es un método biotecnológico efectivo para lograr cambios deseables en el valor nutritivo de las semillas, principalmente por la disminución de algunos factores no nutritivos en legumbres, destacando entre estos el AF; como se observó en el presente trabajo, y el mismo efecto ha sido descrito por otros investigadores (Abdel-Gawad et al., 2016; Gonçalves et al., 2016, Kalpanadevi y Mohan, 2013). Este efecto sobre el AF es debido básicamente a su solubilidad en agua y a la actividad de la enzima fitasa; procesos que se presentan durante el remojo mediante la fitasa constitutiva y continúa con la fitasa inducible durante la germinación (Grenier, 2007). Los resultados obtenidos, superan los porcentajes de disminución descritos por varios investigadores; como Hamed y Hussein (2017), quienes reportaron un $24 \%$ de reducción de AF en Glycine max germinada a TA durante $72 \mathrm{~h}$; Rajni et al. (2016) con un 17,73\% en Phaseolus vulgaris a TA; Mishra y Bhatnagar (2013) con 16,33\% en Vigna unguiculata germinado a TA por 72 h; Rijal y Adhikari (2012) en condiciones experimentales similares a las de este trabajo, reportaron un 35,13\%, de reducción en Glycine max germinada a TA durante cinco días. Estas variaciones en la composición de ciertos compuestos en las semillas, como el ácido fítico y fósforo inorgánico, están relacionadas con las estrategias que establecen las plantas sobre condiciones de germinación, principalmente las características genéticas propias de las semillas, la heterogeneidad de hábitats, estaciones climáticas intrínsecas a su ecosistema (Shaban, 2013; Rijal y Adhikari, 2012). 
Tabla 1. Ácido fítico $(\mathrm{g} / 100 \mathrm{~g})$ en legumbres germinadas a tres temperaturas $\left({ }^{\circ} \mathrm{C}\right) . \mathrm{S} / \mathrm{G}$ : El frijol negro $(P$. vulgaris) no germinó a los dos días a $21^{\circ} \mathrm{C}$, y a ningún tiempo a $29^{\circ} \mathrm{C}$. * El frijol pelón ( $V$. unguiculata) variedad ombligo café, no presentó valores detectables de AF a los cuatro días de germinación en las tres temperaturas $\left(21,25\right.$ y $\left.29^{\circ} \mathrm{C}\right)$. ( ${ }^{\mathrm{a}, \mathrm{b}, \mathrm{c}, \mathrm{d}, \mathrm{d}, \ldots)}$ Medias que tengan la misma letra en cada columna, indica que no son diferentes significativamente $(p<0.05)$.

\begin{tabular}{|c|c|c|c|c|}
\hline \multirow{2}{*}{ Legumbre } & \multirow{2}{*}{$\begin{array}{c}\text { Tiempo de } \\
\text { germinación (días) }\end{array}$} & \multicolumn{3}{|c|}{ Ácido Fítico } \\
\hline & & 21 & 25 & 29 \\
\hline \multirow{4}{*}{ Phaseolus vulgaris } & 0 & $1,86 \pm 0,02^{a}$ & $1,86 \pm 0,02^{a}$ & $S / G$ \\
\hline & 2 & $S / G$ & $0,82 \pm 0,02^{\mathrm{e}}$ & $S / G$ \\
\hline & 4 & $0,35 \pm 0,00^{i}$ & $1,26 \pm 0,06^{c}$ & $\mathrm{~S} / \mathrm{G}$ \\
\hline & 6 & $0,59 \pm 0,02^{g}$ & $0,74 \pm 0,02^{f}$ & $\mathrm{~S} / \mathrm{G}$ \\
\hline \multirow{4}{*}{$\begin{array}{l}\text { Vigna unguiculata, } \\
\text { variedad ombligo } \\
\text { café }\end{array}$} & 0 & $1,12 \pm 0,00^{d}$ & $1,12 \pm 0,00^{d}$ & $1,12 \pm 0,00^{d}$ \\
\hline & 2 & $0,07 \pm 0,00^{m}$ & $0,16 \pm 0,00^{k}$ & $0,13 \pm 0,00^{\prime}$ \\
\hline & 4 & * & * & * \\
\hline & 6 & * & * & * \\
\hline \multirow{4}{*}{$\begin{array}{l}\text { Vigna unguiculata, } \\
\text { variedad corazón } \\
\text { negro }\end{array}$} & 0 & $1,36 \pm 0,02^{b}$ & $1,36 \pm 0,02^{b}$ & $1,36 \pm 0,02^{b}$ \\
\hline & 2 & $0,07 \pm 0,00^{\mathrm{m}}$ & $0,37 \pm 0,01^{i}$ & $0,44 \pm 0,01^{\mathrm{h}}$ \\
\hline & 4 & $0,11 \pm 0,00^{1}$ & $0,25 \pm 0,02^{j}$ & $0,44 \pm 0,00^{h}$ \\
\hline & 6 & $0,12 \pm 0,01^{\prime}$ & $0,16 \pm 0,01^{k}$ & $0,22 \pm 0,00^{j}$ \\
\hline
\end{tabular}

Cabe recordar que, en fuentes de origen vegetal como leguminosas, el ácido fítico es el principal almacén de fósforo, y varios nutrientes con carga están quelados en la molécula del ácido fítico (fitatos), lo que los hace no disponibles para su absorción intestinal, de ahí su bajo aprovechamiento. Es importante subrayar, que el propósito del presente trabajo era encontrar las condiciones adecuadas para que se llevara a cabo la hidrólisis de los fitatos, para obtener fósforo inorgánico libre, así como los nutrientes quelados en él, para aumentar su biodisponibilidad y por consiguiente un mayor aprovechamiento de los mismos, principalmente minerales y proteínas. Dentro de la posible aplicación futura de las harinas obtenidas para la alimentación humana, irá dirigida principalmente a poblaciones vulnerables con deficiencia en proteína y minerales, y no serán adecuadas para individuos con enfermedad renal crónica y enfermedad ósea mineral, donde el exceso de fósforo inorgánico biodisponible es perjudicial (Kalantar-Zadeh, 2013).

\section{Fósforo inorgánico y fósforo total}

Los comportamientos en el contenido de Pi en las legumbres evaluadas fueron similares, detectándose un incremento $(p<0,05)$ conforme avanzó el proceso de germinación (Tabla 2). El contenido de Pi más alto se observó en Vigna unguiculata variedad corazón negro, con $16,36 \mathrm{mg} / \mathrm{g}$ a los seis días de germinación a 29 ${ }^{\circ} \mathrm{C}$, correspondiente a un incremento de 23 veces en relación con el tiempo cero, le siguió Phaseolus vulgaris con un incremento de 16 veces a los cuatro días de germinación a $21^{\circ} \mathrm{C}$. Mientras que, Vigna unguiculata variedad ombligo café tuvo un incremento en Pi de siete veces a los seis días de germinación a $29^{\circ} \mathrm{C}$.

La germinación como proceso biológico propio de todas las semillas, es un proceso multienzimático (Gavindel y Davoodi, 2011) preparativo para llevar a cabo toda la actividad metabólica que requiere generar una nueva planta. Una de las enzimas más importantes en este proceso es la fitasa, que se activa durante el remojo y continua durante la germinación, enzima encargada de llevar a cabo la hidrólisis secuencial del AF o su sal fitato, que mantiene quelados los nutrientes (iones minerales, proteínas y carbohidratos, principalmente) para cuando la semilla los requiera. Dentro de los productos de esta hidrólisis están, inositol fosfato menores (InsP $\mathrm{Ins}_{4}$, y las otras formas), Pi y minerales, estos dos últimos biodisponibles para su aprovechamiento por todos los organismos, principalmente la nueva planta; en caso de las formas menores de inositol fosfato, se considera que tienen un efecto menor como antinutrientes, comparado con el ácido fítico (Abdel-Gawad, 2016).

Por consiguiente, uno de los productos más importantes en la hidrólisis del AF, es la liberación de $\mathrm{Pi}$, el cual se va incrementando conforme avanza el periodo de germinación, como se observó en las legumbres analizadas; encontrándose los valores de Pi más altos, a los seis días de germinación en Vigna unguiculata variedad corazón negro a los $29^{\circ} \mathrm{C}$, siguiéndole Phaseolus vulgaris a $21^{\circ} \mathrm{C}$. Los valores encontrados en este estudio fueron mayores a los descritos por Rijal et al. (2012) para Glycine max bajo condiciones experimentales similares. En relación con el comportamiento de incremento del Pi conforme avanzo el periodo 
de germinación, está de acuerdo con los resultados reportados por Kumar y Sushma (2015); y en desacuerdo con Mahesh et al. (2015), quienes encontraron que el contenido de Pi en semillas de Arachis hipogea y Pisum sativum disminuyó, mientras que en Oryza sativa y Helianthus annuus no presento cambios.

Tabla 2. Fósforo inorgánico $(\mathrm{mg} / \mathrm{g})$ en legumbres germinadas a tres temperaturas $\left({ }^{\circ} \mathrm{C}\right) . \mathrm{S} / \mathrm{G}$ : El frijol negro $(P$. vulgaris) no germinó a los dos días a $21^{\circ} \mathrm{C}$, y a ningún tiempo a $29^{\circ} \mathrm{C}$. (a,b,c,d,...) Medias que tengan la misma letra en cada columna, indica que no son diferentes significativamente $(p<0.05)$.

\begin{tabular}{ccccc}
\hline Legumbre & $\begin{array}{c}\text { Tiempo de } \\
\text { germinación (días) }\end{array}$ & 21 & Fósforo inorgánico & \\
& 0 & $0,94 \pm 0,01^{\mathrm{n}}$ & $0,94 \pm 0,01^{\mathrm{n}}$ & $\mathrm{S} / \mathrm{G}$ \\
Phaseolus vulgaris & 2 & $\mathrm{~S} / \mathrm{G}$ & $0,97 \pm 0,02^{\mathrm{n}}$ & $\mathrm{S} / \mathrm{G}$ \\
& 4 & $15,85 \pm 0,33^{\mathrm{b}}$ & $3,06 \pm 0,14^{\mathrm{l}}$ & $\mathrm{S} / \mathrm{G}$ \\
& 6 & $15,99 \pm 0,04^{\mathrm{b}}$ & $7,65 \pm 0,06^{\mathrm{h}}$ & $\mathrm{S} / \mathrm{G}$ \\
\hline \multirow{2}{*}{$\begin{array}{c}\text { Vigna unguiculata, } \\
\text { variedad ombligo }\end{array}$} & 0 & $1,69 \pm 0,01^{\mathrm{m}}$ & $1,69 \pm 0,01^{\mathrm{m}}$ & $1,69 \pm 0,01^{\mathrm{m}}$ \\
café & 2 & $6,04 \pm 0,09^{\mathrm{i}}$ & $3,79 \pm 0,03^{\mathrm{k}}$ & $8,27 \pm 0,03^{\mathrm{g}}$ \\
& 4 & $7,48 \pm 0,06^{\mathrm{h}}$ & $5,78 \pm 0,01^{\mathrm{i}}$ & $11,07 \pm 0,03^{\mathrm{d}}$ \\
& 6 & $10,29 \pm 0,07^{\mathrm{e}}$ & $8,73 \pm 0,20^{\mathrm{f}}$ & $13,19 \pm 0,18^{\mathrm{c}}$ \\
\hline $\begin{array}{l}\text { Vigna unguiculata, } \\
\text { variedad corazón }\end{array}$ & 0 & $0,67 \pm 0,01^{\mathrm{n}}$ & $0,67 \pm 0,01^{\mathrm{n}}$ & $0,67 \pm 0,01^{\mathrm{n}}$ \\
negro & 2 & $5,23 \pm 0,17^{\mathrm{j}}$ & $0,76 \pm 0,07^{\mathrm{n}}$ & $8,70 \pm 0,13^{\mathrm{f}}$ \\
& 4 & $5,12 \pm 0,13^{\mathrm{j}}$ & $3,92 \pm 0,32^{\mathrm{k}}$ & $13,18 \pm 0,21^{\mathrm{c}}$ \\
\hline
\end{tabular}

Respecto al contenido de Pt en las legumbres evaluadas (Tabla 3), los valores más altos los presentó Vigna unguiculata variedad corazón negro, a los seis días de germinación a 25 y $29^{\circ} \mathrm{C}(\mathrm{p}<0,05)$, con 0,31 veces de incremento $\left(31,62 \%\right.$ de incremento) a $29^{\circ} \mathrm{C}$; mientras que, a $21^{\circ} \mathrm{C}$ los cambios en el contenido de Pt en los diferentes tiempos de germinación fueron menores.

Tabla 3. Fósforo total $(\mathrm{mg} / 100 \mathrm{~g})$ en legumbres germinadas a tres temperaturas $\left({ }^{\circ} \mathrm{C}\right)$. $\mathrm{S} / \mathrm{G}$ : El frijol negro $(P$. vulgaris) no germinó a los dos días a $21^{\circ} \mathrm{C}$, y a ningún tiempo a $29^{\circ} \mathrm{C}$. (a,b,c,d,...) Medias que tengan la misma letra en cada columna, indica que no son diferentes significativamente $(p<0.05)$.

\begin{tabular}{lcccc}
\hline \multicolumn{1}{c}{ Legumbre } & $\begin{array}{c}\text { Tiempo de } \\
\text { germinación (días) }\end{array}$ & 21 & Fósforo total & \\
& 0 & $691 \pm 23^{\mathrm{d}}$ & $691 \pm 23^{\mathrm{d}}$ & $\mathrm{S} / \mathrm{G}$ \\
Phaseolus vulgaris & 2 & $\mathrm{~S} / \mathrm{G}$ & $694 \pm 18^{\mathrm{d}}$ & $\mathrm{S} / \mathrm{G}$ \\
& 4 & $685 \pm 16^{\mathrm{d}}$ & $747 \pm 14^{\mathrm{c}}$ & $\mathrm{S} / \mathrm{G}$ \\
& 6 & $836 \pm 19^{\mathrm{b}}$ & $867 \pm 10^{\mathrm{a}}$ & $\mathrm{S} / \mathrm{G}$ \\
\hline \multirow{2}{*}{ Vigna unguiculata, } & 0 & $593 \pm 0^{\mathrm{h}}$ & $593 \pm 0^{\mathrm{h}}$ & $593 \pm 0^{\mathrm{h}}$ \\
variedad ombligo & 2 & $488 \pm 5^{\mathrm{j}}$ & $636 \pm 5^{\mathrm{f}}$ & $528 \pm 9^{\mathrm{i}}$ \\
café & 4 & $494 \pm 13^{\mathrm{j}}$ & $500 \pm 9^{\mathrm{j}}$ & $617 \pm 28^{\mathrm{g}}$ \\
& 6 & $509 \pm 0^{\mathrm{j}}$ & $596 \pm 23^{\mathrm{h}}$ & $667 \pm 16^{\mathrm{e}}$ \\
\hline \multirow{3}{*}{ Vigna unguiculata, } & 0 & $664 \pm 10^{\mathrm{e}}$ & $664 \pm 10^{\mathrm{e}}$ & $664 \pm 10^{\mathrm{e}}$ \\
variedad corazón & 2 & $602 \pm 0^{\mathrm{h}}$ & $698 \pm 10^{\mathrm{d}}$ & $855 \pm 5^{\mathrm{a}}$ \\
negro & 4 & $651 \pm 14^{\mathrm{e}}$ & $701 \pm 10^{\mathrm{d}}$ & $827 \pm 5^{\mathrm{b}}$ \\
& 6 & $676 \pm 27^{\mathrm{d}}$ & $861 \pm 9^{\mathrm{a}}$ & $873 \pm 14^{\mathrm{a}}$ \\
\hline
\end{tabular}


Al igual que la variedad corazón negro de Vigna unguiculata, Phaseolus vulgaris presento valores similares de $\mathrm{Pt}(\mathrm{p}<0,05)$ a los seis días de germinación a $25^{\circ} \mathrm{C}$, con 0,25 veces de incremento $(25,47 \%$ de incremento). En caso de Vigna unguiculata variedad ombligo café, los cambios observados en el contenido de fósforo total en los diferentes tiempos de germinación a 21 y $29^{\circ} \mathrm{C}$, fueron menores a los de las otras legumbres $(p<0,05)$, mientras que, a $25^{\circ} \mathrm{C}$ no hubo cambios en el contenido de Pt a $25^{\circ} \mathrm{C}$ entre los cero y seis días de germinación.

El contenido de Pt en las legumbres germinadas, presentó porcentajes mayores a los descritos por Araméndiz-Tatis et al. (2016) en los genotipos de frijol caupí analizadas sin procesar, y por Azeque et al. (2011) quienes reportaron un incremento de 5 a $8 \%$ en varias legumbres, incluyendo un Vigna unguiculata; en este mismo estudio esta legumbre presentó los valores más altos de actividad de fitasa y el contenido de AF disminuyó a las 48 h de germinación, comportamiento similar al observado en Vigna unguiculata de este estudio. Los resultados de Pt encontrados en este trabajo difieren con Luo y Xie (2014), quienes reportaron una disminución del Pt en Vicia faba. El aumento en los valores de Pt en las legumbres germinadas, están relacionados con la función que juega el Pi en las células vegetales, principalmente en la construcción de nuevas macromoléculas y estructuras, para generar la nueva planta; actividad metabólica que modifica la materia seca de las semillas germinadas (Kumar y Sushma, 2015).

\section{CONCLUSIONES}

Acorde a los resultados se extraen las siguientes conclusiones principales: 1) la mejor temperatura de germinación para disminuir el ácido fítico fue a $21^{\circ} \mathrm{C}$, siguiéndole $25^{\circ} \mathrm{C}$, durante dos a cuatro días; en esta última temperatura, se observó, el mejor desarrollo de la nueva planta en las legumbres analizadas; 2) el fósforo inorgánico, puede considerarse un buen indicador de la acción de las fitasas endógenas sobre el ácido fítico, ya que se obtuvo el comportamiento esperado durante el proceso de germinación bajo las condiciones experimentales utilizadas; 3) las harinas de las legumbres estudiadas, poseen mayor biodisponibilidad de fósforo inorgánico, y de los nutrientes ligados a él, generando harinas con mayor valor nutricional, factibles de ser utilizadas en la producción de alimentos mejorados, en apoyo a poblaciones que dependen principalmente de la ingesta de alimentos de origen vegetal como fuente de proteína. Sin embargo, no es recomendable utilizar estas harinas en individuos con enfermedad renal crónica y enfermedad ósea mineral, donde el exceso de fósforo inorgánico biodisponible les es perjudicial; y 4) los resultados del presente trabajo aportan conocimiento al enfoque no transgénico de los estudios que se desarrollan para la disminución del AF en semillas, en beneficio de la nutrición de organismos monogástricos y del medio ambiente.

\section{AGRADECIMIENTOS}

Los autores agradecen a las autoridades de la Universidad Juárez Autónoma de Tabasco y al PROMEP, por el financiamiento a los proyectos: UJAT-2009-C05 y UJAT-EXB-130. Así también, al M en C. José Isabel López Naranjo y al MBA. César Omar Hernández Rodríguez, por el apoyo estadístico brindado.

\section{REFERENCIAS}

Abdel-Gawad, A.S., Determination of different inositol phosphate forms in raw, soaked and germinating legumes by anionexchange chromatography, The Pharmaceutical and Chemical Journal, ISSN: 2349-7092 (en línea), 3(1), 183-191 (2016).

Abdel-Gawad, A.S., B.R. Ramadan y R.E.A. Oraby, Legume phytases: Characteristics and changes in activity during germination, International Journal of Agricultural Policy and Research, https://journalissues.org/wpcontent/uploads/2013/06/Abdel-Gawad-et-al.pdf,1 (4), 093-102 (2013)

Araméndiz-Tatis, H., C.E. Cardona-Ayala y E.M. Combatt-Caballero, Contenido nutricional de líneas de frijol caupí (Vigna unguiculata L. Walp) seleccionadas de una población criolla, DOI: 10.4067/S0718-07642016000200007, Información Tecnológica, (en línea), 27(2), 53-60 (2016)

Azeque, M.A., R.M. Elsanhoty, S.J. Egielewa y M.U. Eigbogbo, The effect of germination on the phytase activity, phytate and total phosphorus contents of some Nigerian-grown grain legumes, DOI: 10.1002/jsfa.4150, Journal of the Science of Food and Agriculture, (en línea), 91, 75-79 (2011)

Díaz, M.F., M.A. Martín Cabrejas, A. González, V. Torres y A. Noda, Biotransformación de Vigna unguiculata durante el proceso de germinación, Revista Cubana de Ciencia Agrícola, ISSN: 0034-7485 (en línea), 41(2), $169-174$ (2007). http://www.redalyc.org/articulo.oa?id=193017658013

Gavindel, R.A. y M.G. Davoodi, Evaluation of changes in phytase, $\alpha$-amilase and protease activities of some legume seeds during germination, International Conference on Bioscience, Biochemistry and Bioinformatics, 5, 353-356 (2011)

Gonçalves, A., P. Goufo, A.M. Barros, R. Dominguez-Perles, H. Trindale, E.A.S. Rosa, L. Ferreira, y M. Rodrigues, Cowpea (Vigna unguiculata L. Walp), a renewed multipurpose crop for a more sustainable gri-food system: nutritional advantages and constraints, DOI 10.1002/jsfa.7644, Journal of the Science of Food and Agriculture, (en línea), 96, 29412951 (2016)

Greiner, R. Phytate-degrading enzimes: Regulation of synthesis in microorganisms and plants. DOI: 10.1079/9781845931520.0078, In: Inositol Phosphates: Linking Agriculture and the Environment by B.L. Turner, A.E. Richardson y E.J. Mullaney, Eds., (en línea), pp.78-96 CABI, Wallingford, UK (2007) 
Hamed, I.M. y M.M. Hussein, Influence of processing and fermentation on chemical composition, total phenolic and phytic acid of some cereals and legumes, Research Journal of Pharmaceutical, Biological and Chemical Sciences, ISSN: 09758585 (en línea), 8(1), 335-341 (2017)

Hegeman, C.E. y E.A. Grabau, A novel phytase with sequence similarity to purple acid phosphatases is expressed in cotyledons of germinating soybean seedlings, Plant Physiology, 126, 1598-1608 (2001). https://www.ncbi.nlm.nih.gov/pmc/articles/PMC117159/pdf/pp0801001598.pdf

Jackson, J.F. y H.F. Linskens, Phytic acid in Petunia hybrida pollen is hydrolysed during germination by a phytase. Acta Botanica Neerlandica, 31(5/6), 441-447 (1982). http://natuurtijdschriften.nl/download?type=document;docid=540434

Kalantar-Zadeh K. Patient education for phosphorus management in chronic kidney disease, DOI 10.2147/PPA.S43486 Patient Preference and Adherence, (en línea), 7 379-390 (2013)

Kalpanadevi, V. y V.R. Mohan, Effect of processing on antinutrients and in vitro protein digestibility of the underutilized legume Vigna unguiculata (L.) Walp subsp. unguiculata, DOI 10.1016/j.Iwt.2012.09.030, Food Science and Technology, (en línea), 51(2), 455-461 (2013)

Karp, H., P. Ekholm, V. Kemi, S. Itkonen, T. Hirvonen, S. Narkki y C. Lamberg-Allardt, Differences among total and in vitro digestible phosphorus content of plants foods and beverages, DOI 10.1053/j.jrn.2011.04.004, Journal of Renal Nutrition, (en línea), 22(4), 416-422 (2012)

Kumar, V., A.K. Sinha, H.P.S. Makkar y K. Becker, Dietary roles of phytate and phytase in human nutrition: A review, https://doi.org/10.1016/j.foodchem.2009.11.052, Food Chemistry, (en línea), 120, 945-959 (2010)

Kumar, M. y Sushma, Study of phosphorus profile in indian wheat varieties, International Journal of Science and Research, ISSN: 2319-7064 (en línea), 4(3), 918-922, (2015). www.ijsr.net

Lagunes-Espinoza, L.C., F. Gallardo-López, H. Becerril-Hernández y E.D. Bolaños-Aguilar, Diversidad cultivada y sistema de manejo de Phaseolus vulgaris y Vigna unguiculata en la región de la Chontalpa, Tabasco. Revista Chapingo Serie Horticultura, ISSN 2007-4034 (en línea), 14(1), 13-21 (2008). http://www.scielo.org.mx/pdf/rcsh/v14n1/v14n1a3.pdf

Luo, Y. y W. Xie, Effect of soaking and sprouting on iron and zinc availability in green and white faba vean (Vicia faba L.), DOI: 10.1007/s13197-012-0921-7, Journal of Food Science and Technology, (en línea), 51(12), 3970-3976 (2014)

Mahesh, S., G.J. Pavinthra, M.S. Parvathi, R. Reddy y A.G. Shankar, Effect of processing on phytic acid content and nutrient availability in food grains, International Journal of Agricultural Sciences, ISSN: 2167-0447 (en línea), 5(5), 771 777 (2015). www.internationalscholarsjournals.org

Marrugo-Ligardo, Y.A., P.M. Montero-Castillo y M. Duran-Lengua, Evaluación nutricional de concentrados proteicos de Phaseolus lunatus y Vigna unguiculata, DOI: 10.4067/S0718-07642016000600011, Información Tecnológica, (en línea), 27(6), 107-114 (2016)

Mishra, M. y V. Bhatnagar, Effect of processing methods on phytic acid, total iron and iron bioavailability of cowpea, Asian Journal of Home Science, ISSN 0973-4732 (en línea), 8(1), 51-54 (2013). http://www.researchjournal.co.in/online/AJHS/AJHS-8-1-2013.html

Olukosi, O.A., Biochemistry of phytate and phytases: Applications in monogactric nutrition, 24(2), 58-63 (2012). http://www.bioline.org.br/bk Biokemistri

Rajni, M., K. Mankotia, R. Verma y A. Sandal, Biological protein quality and phytic acid content of domestically processed kidney beans (Phaseolus vulgaris), DOI: 10.18805/ajdfr.v35i4.6631, Asian Journal of Dairy and Food Research, (en línea), 35(4), 315-318 (2016)

Reddy, N.R., S.K. Sathe, D.K. Salunkhe, Phytates in legumes and cereals, https://doi.org/10.1016/S0065-2628(08)60110$X$ Advances in Food Research, (en línea), 28, 1-92 (1982)

Rijal, S. y B. Adhikari, Processing effect on phytic acid and inorganic phosphorus content of soybean (Glycine max, Brown var.), Journal of Food Science and Technology. Nepal, ISSN: 1816-0727 (en línea), 7, $102-106$ (2012). https://www.nepjol.info/index.php/JFSTN/article/.../11208/9066

Rostami, H. y A. Giri, An overview on microbial phytase and its biotechnological applications, International Journal of Advanced Biothechnology and Research, ISSN: 2278-599X (en línea), 4(1), 62-71 (2013). http://www.bipublication.com

Shaban, M. Effect of water and temperature on seed germination and emergence as a seed hydrothermal time model, International Journal of Advanced Biological and Biomedical Research, 1(12), 1686-1691 (2013). http://www.ijabbr.com

Shelton, W.R. y H.J. Harper, A rapid method for the determination of total phosphorus in soil and plant material. lowa State College Journal of Science, 15, 403-413 (1941)

Sotelo, A., J. Mendoza y R.Ma. Argote, Contenido de ácido fítico en algunos alimentos crudos y procesados. Validación de un método colorimétrico, Revista de la Sociedad Química de México, 46(4), $301-306$ (2002). http://www.scielo.org.mx/pdf/rsqm/v46n4/v46n4a2.pdf

Wilcox, J.R., G.S. Premachandra, K.A. Young y V. Raboy, Isolation of high seed inorganic $P$, low-phytate soybean mutants, Crop Science, 40, 1601-1605 (2000). https://pubag.nal.usda.gov/pubag/downloadPDF.xhtml?id=12683\&content=PDF 